\title{
The Relationship Between Interleukin-4 Levels and Cardiovascular Events in Patients with Chronic Kidney Disease
}

This article was published in the following Dove Press journal: Risk Management and Healthcare Policy

\author{
Ling $\mathrm{Gu}^{\prime}$ \\ Huogen Liu (D) ${ }^{\prime}$ \\ Xianhong Liu $^{2}$ \\ Xi Zeng ${ }^{3}$ \\ Zuchen Lei' \\ Xin Wan' \\ 'Department of Critical Care Medicine, \\ Mindong Hospital of Fujian Medical \\ University, Fuan, Fujian 355000, People's \\ Republic of China; ${ }^{2}$ Department of \\ Nephrology, Mindong Hospital of Fujian \\ Medical University, Fuan, Fujian 355000 , \\ People's Republic of China; ${ }^{3}$ Department \\ of Geriatrics, Mindong Hospital of Fujian \\ Medical University, Fuan, Fujian 355000, \\ People's Republic of China
}

Background: Cardiovascular diseases (CVDs) are the main cause of death in patients with chronic kidney disease (CKD). Interleukin-4 (IL-4) is considered an inflammatory cytokine. However, few studies have investigated the association between serum IL-4 and cardiovascular events in CKD. This study investigated whether serum IL-4 levels were associated with an increased risk of cardiovascular (CV) events in patients with CKD.

Patients and Methods: A total of 302 patients with stage 1-5 CKD were followed up for a mean of 32 (range=4-36) months for end points (CV events). Serum IL-4 levels were measured at baseline. The independent relationship between serum IL-4 and the risk of CV events was assessed with multivariate Cox regression analysis.

Results: The average age of this cohort $(\mathrm{N}=302)$ was 65.4 years. A total of $69.9 \%$ of them were male. CV events numbered 41 (13.6\%) during the follow-up period. The Kaplan-Meier analysis showed that the rate of CV events was higher in patients with CKD with IL-4 levels above the mean $(126.2 \mathrm{pg} / \mathrm{mL})$ than in those with IL-4 levels below the mean. The multivariate Cox proportional hazard analysis revealed that serum IL-4 (HR=1.650, 95\% CI 1.266-2.210, $P<0.001)$ was associated with $\mathrm{CV}$ events in these patients with $\mathrm{CKD}$. Sensitivity analysis showed that the association between serum IL-4 and CV events was not affected by the use of anti-inflammatory medication. The significant association between higher IL-4 levels and increased risk of CV events existed in patients with CKD3-5 but not in patients with CKD1-2 by using the stratified analysis.

Conclusion: Higher serum IL-4 levels were associated with an increased risk of CV events during follow-up. Elevated serum IL-4 levels may help clinicians predict early CV events in patients with CKD.

Keywords: interleukin-4, chronic kidney disease, cardiovascular event, Cox regression, prognosis

\section{Introduction}

Chronic kidney disease (CKD) has been recognized as a public health problem and is an increasingly prevalent condition that has affected $10-12 \%$ of the general population worldwide. ${ }^{1,2}$ The incidence rate of cardiovascular diseases (CVDs) in patients with CKD is significantly higher than that in non-CKD patients, which has been the main cause of death in patients with CKD. ${ }^{3-5}$ Although the exact mechanisms explaining this association are still unclear, increasing inflammation and oxidative stress in CKD are considered the primary mechanisms leading to an increased risk of cardiovascular (CV) events. ${ }^{6,7}$ Early prediction of CVDs is of
Correspondence: Xin Wan

Department of Critical Care Medicine, Mindong Hospital of Fujian Medical

University, No. 89 Heshan Road, Fuan

City 355000, Fujian Province, People's

Republic of China

Tel +8615259367910

Emailwancy505@I63.com
Risk Management and Healthcare Policy 2020:13 237I-2377

submit your manuscript (c) (i) $2020 \mathrm{Gu}$ et al. This work is published and licensed by Dove Medical Press Limited. The full terms of this license are available at https://www.dovepress.com/terms.php cc) ${ }_{\mathrm{BY}} \mathrm{NC}_{\text {and }}$ ancorporate the Creative Commons Attribution - Non Commercial (unported, v3.0) License (http://creativecommons.org/licenses/by-nc/3.0/). By accessing the work you hereby accept the Terms. Non-commercial uses of the work are permitted without any further permission from Dove Medical Press Limited, provided the work is properly attributed. For permission for commercial use of this work, please see paragraphs 4.2 and 5 of our Terms (https://www.dovepress.com/terms.php). 
great significance to help clinicians intervene in the disease in a timely manner and improve the prognosis of patients with CKD.

Chronic inflammation plays one of the most prominent roles in CKD. Chronic inflammatory status exists in most CKD populations and is accompanied by a decline in renal function with an increased inflammatory response. ${ }^{8}$ Previous studies have suggested that most patients with CKD stages ${ }^{3-5}$ have increased levels of serum C-reactive protein (CRP). ${ }^{9,10}$ The highest serum CRP levels were found in patients with dialysis. ${ }^{11}$ In patients with $\mathrm{CKD}$, systemic inflammation is associated with increased mortality and poor quality of life, mainly due to CVDs that are also related to a chronic inflammatory state. ${ }^{12}$ Among the known inflammatory biomarkers, interleukin (IL)-6 has been shown to have a good predictive function of $\mathrm{CV}$ outcomes in CKD. ${ }^{13}$ Emerging studies support that persistent inflammation plays a major role in renal fibrosis and functional decline, ${ }^{14}$ promoting the progression of CKD. ${ }^{15}$ Recently, some studies have also shown the key role of interleukin (IL-4) in inflammation, which is a new field of inflammatory research. IL-4 can inhibit the production of proinflammatory cytokines, including tumor necrosis factor- $\alpha$ (TNF- $\alpha$ ), interleukin-1 (IL-1), IL-6 and prostaglandin E2 (PGE2). Additionally, IL-4 can also upregulate the expression of anti-inflammatory mediators such as IL1 type II receptors and IL-1 receptor antagonists. ${ }^{16,17}$

Until now, however, few studies have suggested that serum IL-4 was associated with CV events in patients with CKD. Given the important role of IL-4 in the regulation of inflammation, we hypothesized that IL-4 may be associated with CV outcomes in patients with CKD. The aim of this study was to investigate whether increased serum IL-4 levels were associated with a higher risk of $\mathrm{CV}$ events.

\section{Patients and Methods}

\section{Study Population}

A total of 302 patients with CKD from the National Population Health Science Data Center in China were studied between May 2014 and August 2016. This database contains enough clinical data of patients from major hospitals in China. In this study, all patients were stable for more than 6 months without any acute CVDs before their admission. All patients with CKD were prospectively followed up for a mean of 32 (range=4-36) months for fatal and nonfatal $\mathrm{CV}$ events after discharge. According to the
Kidney Disease: Improving Global Outcomes guidelines, "CKD is defined as abnormalities of kidney structure or function, present for $>3$ months,". 18 Patients who were diagnosed with CKD were included in the study. For the purpose of this study, CKD1-2 was regarded as the early stage and CKD3-5 as the middle and late stage in all CKD patients. Patients with a history of neoplastic diseases or other serious diseases were excluded. Eleven patients were excluded from the study due to severe liver or lung diseases and malignant diseases.

The causes of CKD, including primary kidney disease, hypertension, diabetes mellitus, coronary heart disease, nephrolithiasis and others, were based on the patient's current or previous medical records. Clinical characteristics, including age, sex, body mass index (BMI), current smoker, current drinker, admission systolic blood pressure (SBP) and diastolic blood pressure (DBP), hyperlipidemia and dialysis history, were collected from patient interviews or medical records. According to the Declaration of Helsinki guidelines, the Ethics Committee of the affiliated Mindong Hospital of Fujian Medical University approved this study, and all patients gave written informed consent.

\section{Follow-Up}

All patients with CKD were prospectively followed up for a mean of 32 (range=4-36) months. Patients were followed up by reviewing the medical records or telephone 4 times every year until the appearance of CV events. The end points ( $\mathrm{CV}$ events) in this study included myocardial infarction, stroke, heart failure (HF) and CVD death. CVD death was defined as death due to myocardial infarction, stroke or progressive HF confirmed by two cardiologists.

\section{Measurement of IL-4}

Fasting venous blood samples were collected from patients with CKD in the early morning after admission. The samples were prepared immediately by centrifugation and processed for the determination of IL-4 levels. Serum IL-4 levels were measured by using enzyme-linked immunosorbent assay (ELISA; Fujirebio Diagnostics, Malvern, Pennsylvania). This ELISA allows accurate measurements of serum IL-4 levels and has a detection limit of $\leq 5 \mathrm{pg} / \mathrm{mL}$ and a coefficient of variance $(\mathrm{CV}) \leq 15 \%$. The interassay reproducibility was $97.7 \%$.

\section{Laboratory Measurements and Definition}

The venous blood samples collected from these patients with $\mathrm{CKD}$ in the early morning after admission were also used to 
test other serum indicators. Routine laboratory measurements were performed, including hemoglobin $(\mathrm{Hb})$, highdensity lipoprotein (HDL), low-density lipoprotein (LDL), fasting blood glucose (FBG), hypersensitive C-reactive protein (hs-CRP), glycated hemoglobin (HbAlc), albumin (ALB), serum creatinine ( $\mathrm{SCr}$ ), urea nitrogen (BUN), and uric acid (UA), by using the Roche Modular system (Roche, Mannheim, Germany). N-Terminal prohormone of B-type natriuretic peptide (NT-proBNP) levels were measured by immunoassay on an ELECSYS2010 instrument (ELECSYS proBNP, Roche Diagnostics, Germany). Body mass index (BMI) was calculated by dividing weight by height squared. The simplified Modification of Diet in Renal Disease formula was used to calculate eGFR. ${ }^{19}$

\section{Statistical Analyses}

All of the data were analyzed by using SPSS 23.0. The Mann-Whitney $U$-test was used to analyze data that were not normally distributed and expressed as the median (interquartile range $[\mathrm{IQR}])$. Independent t-tests were used to analyze normally distributed data that were presented as the mean \pm standard deviation (SD). The chi-square test was used for analyzing categorical variables. Serum IL-4 levels were categorized by quartiles (quartile $4: \geq 75$ th percentile, quartile $3: \geq 50$ th to 75 th percentile, quartile $2: \geq 25$ th to 50 th percentile, quartile $1:<25$ th percentile). Cox proportional hazard analysis was used to identify the independent prognostic factors for $\mathrm{CV}$ events in patients with $\mathrm{CKD}$. To further examine the association between IL-4 and CV events, we excluded the effect of anti-inflammatory medication on the correlation by sensitivity analysis. Additionally, we also analyzed the association between IL-4 levels at baseline and CV events after a follow-up of 3 years stratified by CKD stage (CKD1-2 and CKD3-5). According to the Kaplan-Meier method, CV event-free curves were constructed and compared using the Log rank test. $P \leq 0.05$ was considered to be statistically significant.

\section{Results}

\section{Clinical Characteristics at Baseline}

The clinical characteristics of patients with CKD are presented in Table 1. In these patients with CKD, the average age was $65.4 \pm 7.9$ years; $211(69.9 \%)$ of them were male; $54(17.9 \%)$ were current smokers; 39 (12.9\%) were current drinkers; and 122 (40.4\%) had a dialysis history. The mean level of IL-4 was $126.2 \mathrm{pg} / \mathrm{mL}$, and $\mathrm{CV}$ events were identified in $41(13.6 \%)$ patients with CKD.
Table I Clinical Characteristics at Baseline

\begin{tabular}{|c|c|}
\hline Characteristics & Total $(\mathrm{N}=302)$ \\
\hline Age $(\mathrm{Y})$ & $65.4 \pm 7.9$ \\
\hline Male, n (\%) & $211(69.9)$ \\
\hline Admission SBP (mm Hg) & $129.7(\mid 06.5-146.8)$ \\
\hline Admission DBP (mm Hg) & $86.3(72.7-96.4)$ \\
\hline Current smoker, n (\%) & $54(17.9)$ \\
\hline Current drinker, n (\%) & $39(12.9)$ \\
\hline BMI $\left(\mathrm{kg} / \mathrm{m}^{2}\right)$ & $27.8 \pm 4.81$ \\
\hline Hyperlipidemia, n (\%) & $46(15.2)$ \\
\hline Dialysis history & $122(40.4)$ \\
\hline Anti-inflammatory medication & $137(45.4)$ \\
\hline CV events & $4 \mid(13.6)$ \\
\hline \multicolumn{2}{|l|}{ Causes of CKD } \\
\hline Primary kidney disease, n (\%) & $125(4 \mid .4)$ \\
\hline Hypertension, n (\%) & $121(40.1)$ \\
\hline Diabetes mellitus, n (\%) & $77(25.5)$ \\
\hline Coronary heart disease, n (\%) & $16(5.3)$ \\
\hline Nephrolithiasis, n (\%) & $43(14.2)$ \\
\hline Others, n (\%) & $13(4.3)$ \\
\hline \multicolumn{2}{|l|}{ Laboratory measurements } \\
\hline $\mathrm{IL}-4(\mathrm{pg} / \mathrm{mL})$ & $126.2 \pm 28.7$ \\
\hline eGFR $\left(\mathrm{mL} / \mathrm{min} / \mathrm{l} .73 \mathrm{~m}^{2}\right)$ & $76.4(49.3-85.9)$ \\
\hline $\mathrm{SCr}$ (ummol/L) & $171.0(\mid 36.0-288.0)$ \\
\hline BUN (mmol/L) & $9.7(7.4-12.3)$ \\
\hline UA (ummol/L) & $466.0(4 \mid 2.5-678.0)$ \\
\hline NT-proBNP (pg/mL) & 478.I (237.4-66I.8) \\
\hline $\mathrm{Hs}-\mathrm{CRP}$ (mg/L) & $3.5(0.7-9.9)$ \\
\hline $\mathrm{Hb}(\mathrm{g} / \mathrm{L})$ & II4.0 (96.0-129.0) \\
\hline $\mathrm{HDL}(\mathrm{mmol} / \mathrm{L})$ & $0.94 \pm 0.13$ \\
\hline $\mathrm{LDL}(\mathrm{mmol} / \mathrm{L})$ & $2.43 \pm 0.74$ \\
\hline $\operatorname{ALB}(g / L)$ & $35.3(31.6-43.7)$ \\
\hline $\mathrm{FBG}(\mathrm{mmol} / \mathrm{L})$ & $5.72(4.53-8.26)$ \\
\hline HbAlc (\%) & $5.7 \pm 1.2$ \\
\hline \multicolumn{2}{|l|}{ CKD stage } \\
\hline CKDI-2 & $192(63.6)$ \\
\hline CKD3-5 & $110(36.4)$ \\
\hline
\end{tabular}

Notes: Data are presented as mean \pm SD for normally distributed data, as median (interquartile range) for nonnormally distributed data, and as $n$ (\%) for categoric variables.

Abbreviations: SBP, systolic blood pressure; DBP, diastolic blood pressure; BMI, body mass index; CV, cardiovascular; CKD, chronic kidney disease; IL-4, interleukin 4; eGFR, estimated glomerular filtration rate; $\mathrm{SCr}$, serum creatinine; $\mathrm{BUN}$, urea nitrogen; UA, uric acid; NT-proBNP, N-terminal prohormone of B-type natriuretic peptide; Hs-CRP, hypersensitive C-reactive protein; $\mathrm{Hb}$, hemoglobin; $\mathrm{HDL}$, high density lipoprotein; LDL, low density lipoprotein; ALB, albumin; FBG, fasting blood glucose; HbAlc, glycated hemoglobin.

\section{Serum IL-4 Levels Were Independently Associated with CV Events in Patients with CKD}

All included patients $(\mathrm{N}=302)$ were prospectively followed up for a mean of 32 (range=4-36) months. CV events 
Table 2 Multivariate Cox Regression Analysis of Predicting CV Events in 302 Patients with CKD

\begin{tabular}{|l|l|l|l|}
\hline Serum IL-4 Levels & Model I & Model 2 & Model 3 \\
\hline Quartile I (low) & 1.000 (ref.) & 1.000 (ref.) & 1.000 (ref.) \\
Quartile 2 & $1.213(1.016-1.621)$ & $1.171(1.010-1.598)$ & $1.103(1.001-1.427)$ \\
Quartile 3 & $1.382(1.128-1.853)$ & $1.352(1.167-1.761)$ & $1.305(1.112-1.682)$ \\
Quartile 4 (high) & $1.828(1.331-2.797)$ & $1.749(1.304-2.544)$ & $1.650(1.266-2.210)$ \\
P-trend & $<0.001$ & $<0.001$ & $<0.001$ \\
\hline
\end{tabular}

Notes: Model I: Adjusted for age and gender. Model 2: Adjusted for age, gender, BMI, current smoker, current drinker, admission SBP and DBP, hyperlipidemia, dialysis history and causes of CKD. Model 3: Adjusted for age, gender, BMI, current smoker, current drinker, admission DBP and DBP, hyperlipidemia, dialysis history, causes of CKD and laboratory measurements.

Abbreviations: CKD, chronic kidney disease; CV, cardiovascular; IL-4, interleukin-4; BMI, body mass index; SBP, systolic blood pressure; DBP, diastolic blood pressure.

occurred in 41 of the patients with CKD. Thirty-three events requiring rehospitalization occurred due to myocardial infarction $(\mathrm{N}=16)$, stroke $(\mathrm{N}=11)$ and progressive $\mathrm{HF}$ $(\mathrm{N}=6)$ during the follow-up period. Ten patients with $\mathrm{CKD}$ died in which 7 of these deaths were caused by $\mathrm{CV}$ events, including myocardial infarction $(\mathrm{n}=4)$, stroke $(\mathrm{N}=2)$ and progressive HF $(\mathrm{n}=1)$.

To determine the association of serum IL-4 levels with $\mathrm{CV}$ events in patients with $\mathrm{CKD}$, a multivariate Cox proportional hazard regression model was performed (Table 2). Multivariate Cox proportional hazard analysis revealed that IL-4 (HR=1.650, 95\% CI 1.266-2.210, $P<0.001)$ was an independent prognostic factor for $\mathrm{CV}$ events after adjusting for age, sex, BMI, current smoker, current drinker, admission
DBP and DBP, hyperlipidemia, dialysis history, causes of CKD and laboratory measurements. Kaplan-Meier analysis suggested that patients with CKD with serum IL-4 levels above the mean $(126.2 \mathrm{pg} / \mathrm{mL})$ had a higher rate of CV events than patients with serum IL-4 levels below the mean value (Log rank test, $P<0.001$ ) (Figure 1).

We performed an additional sensitivity analysis by adding anti-inflammatory medication as a covariate to assess the association between serum IL- 4 and CV events in patients with CKD. The multivariate Cox analysis showed that higher IL-4 levels were still independently associated with a higher risk of $\mathrm{CV}$ events after adjusting for related confounding factors (Table 3). Similarly, compared with patients who had IL-4 levels below the mean

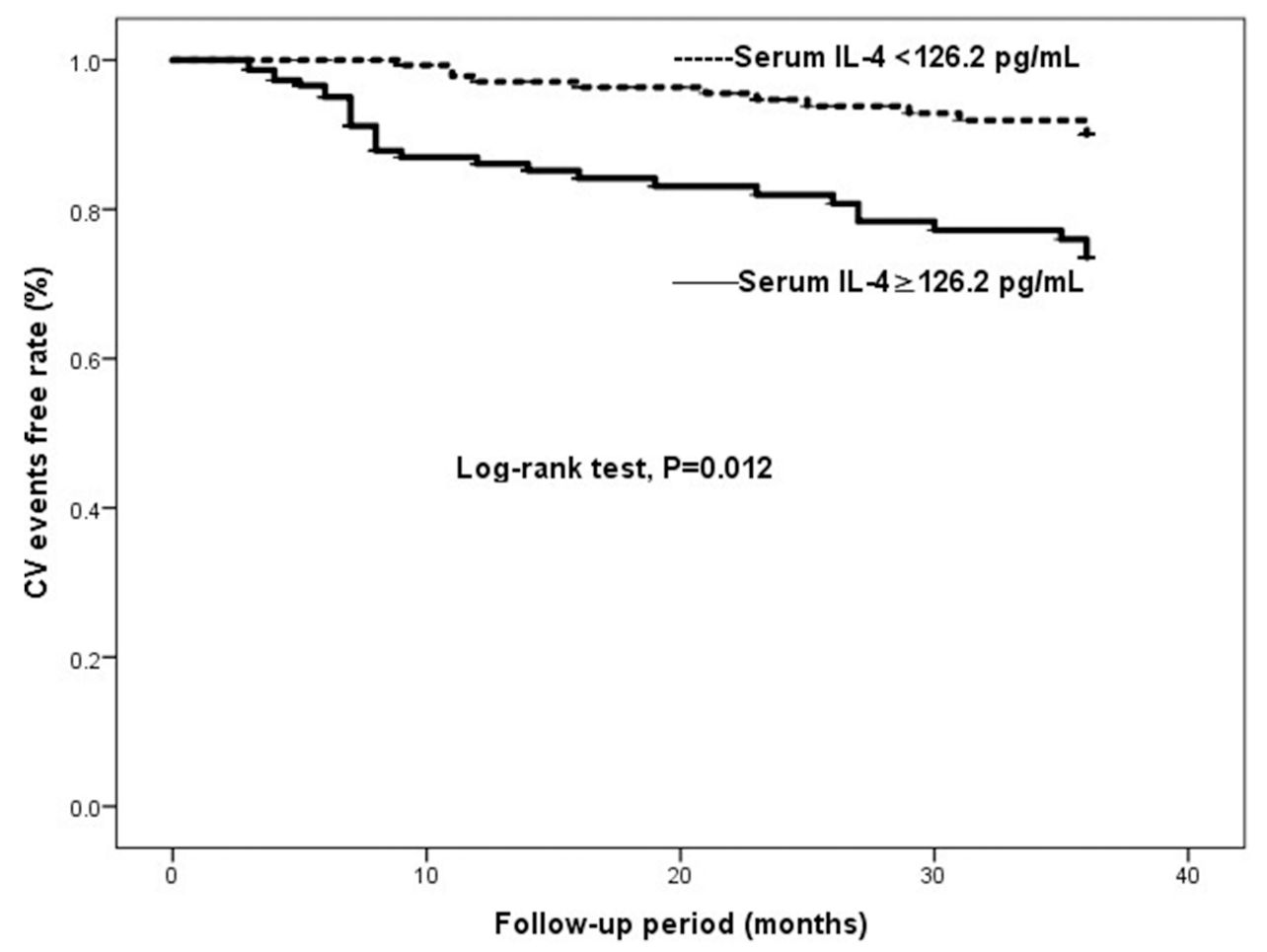

Figure I Kaplan-Meier analysis of cardiovascular (CV) events free stratified into 2 groups by the mean level of serum interleukin-4 (IL-4). 
Table 3 Sensitivity Analysis by Adding Anti-Inflammatory Medication as Covariate for Predicting CV Events in 302 Patients with CKD by Multivariate Cox Regression Analysis

\begin{tabular}{|l|l|l|l|}
\hline Serum IL-4 Levels & Model I & Model 2 & Model 3 \\
\hline Quartile I (low) & 1.000 (ref.) & 1.000 (ref.) & 1.000 (ref.) \\
Quartile 2 & $1.201(1.012-1.565)$ & $1.130(1.008-1.523)$ & $1.071(1.001-1.395)$ \\
Quartile 3 & $1.284(1.113-1.727)$ & $1.254(1.100-1.702)$ & $1.244(1.072-1.631)$ \\
Quartile 4 (high) & $1.820(1.246-2.294)$ & $1.742(1.210-2.136)$ & $1.547(1.196-2.056)$ \\
P-trend & $<0.001$ & 0.002 & 0.007 \\
\hline
\end{tabular}

Notes: Model I: Adjusted for age, gender and anti-inflammatory medication. Model 2: Adjusted for age, gender, BMI, current smoker, current drinker, admission SBP and DBP, hyperlipidemia, dialysis history, causes of CKD and anti-inflammatory medication. Model 3: Adjusted for age, gender, BMI, current smoker, current drinker, admission $\mathrm{DBP}$ and DBP, hyperlipidemia, dialysis history, causes of CKD, laboratory measurements and anti-inflammatory medication.

Abbreviations: CKD, chronic kidney disease; CV, cardiovascular; IL-4, interleukin-4; BMI, body mass index; SBP, systolic blood pressure; DBP, diastolic blood pressure.

value, Kaplan-Meier survival curves still showed that the rate of CV events was higher in patients with IL-4 levels above the median (Log rank test, $P<0.001$ ) (data not shown).

\section{Serum IL-4 Levels Were Independently Associated with CV Events in Patients with CKD by Stratified Analysis}

The incidence of cardiovascular complications is related to the severity of CKD. Therefore, all patients with CKD were divided into two groups (CKD1-2 group and CKD3-5 group) according to CKD stage (Table 4). Our results showed that serum IL-4 levels were independently associated with $\mathrm{CV}$ events in the CKD3-5 group but not in the CKD1-2 group, which implies that IL-4 may be a better serum marker for predicting $\mathrm{CV}$ events in patients with end-stage CKD.

\section{Discussion}

Our findings in this study suggested that serum IL-4 levels were independently related to an increased risk of $\mathrm{CV}$ events in patients with CKD by using multivariate Cox proportional hazard analysis. Kaplan-Meier survival curves suggested that the rate of $\mathrm{CV}$ events was higher in patients with CKD with IL-4 levels above the median $(126.2 \mathrm{pg} / \mathrm{mL})$ than in those patients with serum IL-4 levels below the median value. The association between higher IL-4 levels and increased CV events was affected by CKD stage using stratified analysis.

CKD is a chronic inflammatory disease characterized by infiltration of inflammatory cells and renal interstitial fibrosis. $^{20,21}$ The pathophysiology involved in the development of chronic inflammation in CKD has not yet been completely elucidated. However, it is clear that the more chronic renal fibrosis there is, the higher the level of the inflammatory response. The incidence rate of CVDs in patients with CKD is significantly higher than that in nonCKD patients, which has been the main cause of death in patients with $\mathrm{CKD}^{3-5,22}$ Disproportionately increasing inflammation and oxidative stress play an important role in the occurrence and development of atherosclerosis, which are believed to be responsible for a significant increase in the risk of CVD death in patients with CKD. ${ }^{6,7}$

Existing studies show that the role of IL-4 in inflammation is contradictory. Some studies have shown that IL-4 has anti-inflammatory properties, ${ }^{16,17}$ while others have suggested that IL-4 has a key role in regulating the differentiation of inflammatory cells such as B and $\mathrm{T}$ cells, ${ }^{23}$ which produce other proinflammatory cytokines

Table 4 Multivariate Cox Regression Analysis of Predicting CV Events in 302 Patients with CKD Stratified by CKD Stage

\begin{tabular}{|l|l|l|l|}
\hline CKD Stage & Model I & Model 2 & Model 3 \\
\hline CKDI-2 & $1.210(1.012-1.598)$ & $1.162(1.009-1.522)$ & $1.107(1.001-1.415)$ \\
P value & 0.015 & 0.026 & 0.053 \\
CKD3-5 & $2.521(1.640-3.798)$ & $2.389(1.616-3.494)$ & $2.288(1.401-3.110)$ \\
P value & $<0.001$ & $<0.001$ & $<0.001$ \\
\hline
\end{tabular}

Notes: Model I: Adjusted for age and gender. Model 2: Adjusted for age, gender, BMI, current smoker, current drinker, admission SBP and DBP, hyperlipidemia, dialysis history, anti-inflammatory medication, and causes of CKD. Model 3: Adjusted for age, gender, BMI, current smoker, current drinker, admission DBP and DBP, hyperlipidemia, dialysis history, anti-inflammatory medication, causes of CKD and laboratory measurements.

Abbreviations: CKD, chronic kidney disease; CV, cardiovascular; IL-4, interleukin-4; BMI, body mass index; SBP, systolic blood pressure; DBP, diastolic blood pressure. 
and play an important role in the humoral immune response. $^{24}$ It has also been reported that IL-4 signaling pathway transduction plays a proinflammatory role in inflammatory responses. ${ }^{25}$ IL-4 can also induce complex inflammatory responses, including stimulating the secretion of peripheral blood neutrophils, promoting the expression of IL- 8 and TNF- $\alpha$, and inhibiting the expression of IL-1 $\beta .^{26,27}$ However, few studies have investigated the association between serum IL-4 levels and CV events in patients with CKD. In this study, we found that high serum IL-4 levels were independently associated with increased risk of $\mathrm{CV}$ events in patients with $\mathrm{CKD}$, which is consistent with previous research on the mechanisms by which IL-4 plays an important role in proinflammatory responses.

This study has some notable strengths. On the one hand, we first found that serum IL-4 may be considered an effective prognostic factor for $\mathrm{CV}$ outcomes in patients with CKD. It is important for us to identify risk factors for $\mathrm{CV}$ events and use them as targets for therapy or predictors for prognosis, which would make it possible to treat and prevent CVDs in patients with CKD. Our results showed that higher serum IL-4 levels in patients with CKD had a higher risk of $\mathrm{CV}$ events. On the other hand, we ensured comprehensive follow-up and rigorous adjudication of $\mathrm{CV}$ events. The IL-4 assay chosen for this analysis is both widely available and analytically stable.

\section{Limitations}

This study has several limitations. First, the study included a small sample size. Large-scale prospective cohort studies and randomized controlled trials are needed to better understand the association between serum IL-4 and CV events in patients with CKD. Second, some inflammatory cytokines, including IL-1, IL-6, TNF- $\alpha$ and others associated with IL-4, should be included in this study to identify the prognostic value of these inflammatory markers for predicting CV events.

\section{Conclusions}

Elevated serum IL-4 was significantly and independently associated with a higher rate of $\mathrm{CV}$ events in patients with CKD during a follow-up of 3 years and may be a reliable prognostic factor for predicting $\mathrm{CV}$ events in patients with CKD. Elevated serum IL-4 levels may help clinicians predict early $\mathrm{CV}$ events in patients with CKD. there is no funding to report

\section{Funding}

There is no funding to report.

\section{Disclosure}

The authors report no conflicts of interest for this work.

\section{References}

1. Levin A, Tonelli M, Bonventre J, et al. Global kidney health 2017 and beyond: a roadmap for closing gaps in care, research, and policy. Lancet. 2017;390:1888-1917. doi:10.1016/S0140-6736(17)30788-2

2. Kelsey R. Cardiovascular disease: chronic kidney disease: a risk factor for stroke and bleeding in patients with atrial fibrillation. Nat Rev Nephrol. 2012;8(10):551. doi:10.1038/nrneph.2012.168

3. Foley RN, Murray AM, Li S. Chronic kidney disease and the risk for cardiovascular disease, renal replacement, and death in the United States medicare population, 1998 to 1999. J Am Soc Nephrol. 2005;16(2):489-495. doi:10.1681/ASN.2004030203

4. Levin A, Djurdjev O, Barrett B, et al. Cardiovascular disease in patients with chronic kidney disease: getting to the heart of the matter. $\mathrm{Am}$ J Kidney Dis. 2001;38(6):1398-1407. doi:10.1053/ajkd.2001.29275

5. Ninomiya T, Kiyohara Y, Kubo M, et al. Chronic kidney disease and cardiovascular disease in a general Japanese population: the Hisayama study. Kidney Int. 2005;68(1):228-236. doi:10.1111/ j.1523-1755.2005.00397.x

6. Cachofeiro V, Goicochea M, de Vinuesa SG, et al. Oxidative stress and inflammation, a link between chronic kidney disease and cardiovascular disease. Kidney Int. 2008;74(Suppl 111):S4-S9.

7. Naito K, Anzai T, Yoshikawa T, et al. Impact of chronic kidney disease on postinfarction inflammation, oxidative stress, and left ventricular remodeling. $J$ Card Fail. 2008;14(10):0-838. doi:10.1016/j.cardfail.2008.07.233

8. Gupta J, Mitra N, Kanetsky PA, et al. Association between albuminuria, kidney function, and inflammatory biomarker profile in CKD in CRIC. Clin J Am Soc Nephrol. 2012;7:1938-1946. doi:10.2215/ CJN.03500412

9. Eustace JA, Astor B, Muntner PM, et al. Prevalence of acidosis and inflammation and their association with low serum albumin in chronic kidney disease. Kidney Int. 2004;65:1031-1040. doi:10.1111/j.1523-1755.2004.00481.x

10. Stenvinkel P, Wanner C, Metzger T, et al. Inflammation and outcome in end-stage renal failure: does female gender constitute a survival advantage? Kidney Int. 2002;62:1791-1798. doi:10.1046/j.15231755.2002.00637.x

11. Carrero JJ, Stenvinkel P. Inflammationin end-stage renal diseasewhat have welearnedin10 years? Semin Dial. 2010;23:498-509. doi:10.1111/j.1525-139X.2010.00784.X

12. Jankowska M, Cobo G, Lindholm B, et al. Inflammation and proteinenergy wasting intheuremicmilieu. Contrib Nephrol. 2017;191:58-71.

13. Sun J, Axelsson J, Machowska A, et al. Biomarkers of cardiovascular disease and mortality risk in patients with advanced CKD. Clin J Am Soc Nephrol. 2016;11:1163-1172. doi:10.2215/CJN.10441015

14. Kooman JP, Kotanko P, Schols AM, et al. Chronic kidney disease and premature ageing. Nat Rev Nephrol. 2014;10:732-742.

15. Amdur RL, Feldman HI, Gupta J, et al. Inflammation and progression of CKD: the CRIC study. Clin $J$ Am Soc Nephrol. 2016;11:1546-1556. doi:10.2215/CJN.13121215

16. Ouyang BS, Che JL, Gao J, et al. [Effects of electroacupuncture and simple acupuncture on changes of IL-1, IL-4, IL-6 and IL-10 in peripheral blood and joint fluid in patients with rheumatoid arthritis]. Zhongguo Zhen Jiu. 2010;30:840-844.Chinese.

17. Ursaciuc C, Surcel M, Ciotaru D, et al. Regulatory T cells and TH1/ TH2 cytokines as immunodiagnosis keys in systemic autoimmune diseases. Roum Arch Microbiol Immunol. 2010;69:79-84. 
18. Andrassy KM. Comments on 'KDIGO 2012 clinical practice guideline for the evaluation and management of chronic kidney disease'. Kidney Int. 2013;84(3):622-623. doi:10.1038/ki.2013.243

19. Levey AS, Stevens LA, Schmid $\mathrm{CH}$, et al. A new equation to estimate glomerular filtration rate. Ann Intern Med. 2009;150 (9):604-612. doi:10.7326/0003-4819-150-9-200905050-00006

20. Xiao X, Du C, Yan Z, et al. Inhibition of necroptosis attenuates kidney inflammation and interstitial fibrosis induced by unilateral ureteral obstruction. Am J Nephrol. 2017;46(2):131-138. doi:10. $1159 / 000478746$

21. Hanatani S, Izumiya Y, Araki S, et al. Abstract 11021: akt1-mediated skeletal muscle growth attenuates renal interstitial fibrosis and inflammation in obstructive nephropathy. circulation. 2012;A11021.

22. Yuhao S, Ying $H$, Ying $J$, et al. The association between serum retinol-binding protein 4 levels and cardiovascular events in patients with chronic kidney disease. Lab Med. 2020.

23. Nelms K, Keegan AD, Zamorano J, et al. The IL-4 receptor: signaling mechanisms and biologic functions. Annu Rev Immunol. 1999;17:701-738. doi:10.1146/annurev.immunol.17.1.701
24. Mosmann TR, Coffman RL. TH1 and TH2 cells: different patterns of lymphokine secretion lead to different functional properties. Annu Rev Immunol. 1989;7:145-173. doi:10.1146/annurev.iy.07.040189. 001045

25. Guenova E, Hoetzenecker W, Hoerber S, et al. Systemic IL-4 treatment suppresses IL-23 and abrogates inflammatory responses in T cell mediated delayed type hypersensitivity. J Investig Dermatol. 2010.

26. Bao L, Shi VY, Chan LS. IL-4 up-regulates epidermal chemotactic, angiogenic, and pro-inflammatory genes and down-regulates antimicrobial genes in vivo and in vitro: relevant in the pathogenesis of atopic dermatitis. Cytokine. 2013;61:419-425. doi:10.1016/j. cyto.2012.10.031

27. Lavoie-Lamoureux A, Moran K, Beauchamp G, et al. IL-4 activates equine neutrophils and induces a mixed inflammatory cytokine expression profile with enhanced neutrophil chemotactic mediator release ex vivo. Am J Physiol Lung Cell Mol Physiol. 2010;299: L472-82. doi:10.1152/ajplung.00135.2009
Risk Management and Healthcare Policy

\section{Publish your work in this journal}

Risk Management and Healthcare Policy is an international, peerreviewed, open access journal focusing on all aspects of public health, policy, and preventative measures to promote good health and improve morbidity and mortality in the population. The journal welcomes submitted papers covering original research, basic science, clinical \& epidemiological studies, reviews and evaluations, guidelines, expert opinion and commentary, case reports and extended reports. The manuscript management system is completely online and includes a very quick and fair peer-review system, which is all easy to use. Visit http://www.dovepress.com/testimonials.php to read real quotes from published authors. 